\title{
Hipotensão pós-exercício induzida por treinamento aeróbio, de força e concorrente: aspectos metodológicos e mecanismos fisiológicos
}

\author{
Post-exercise hypotension induced by aerobic, strength and concurrent \\ training: methodological issues and underlying physiological mechanisms
}

\author{
Felipe A. Cunha \\ Lenifran Matos-Santos \\ Renato O. Massaferri \\ Tainah P. Lima Monteiro \\ Paulo T. V. Farinatti ${ }^{\star}$
}

\section{Resumo}

Evidências indicam que reduções crônicas na pressão arterial (PA) provocadas por exercício físico dependem, em grande medida, da capacidade de se induzir após cada sessão de treinamento o fenômeno da hipotensão pós-exercício (HPE). A presente revisão descreve artigos sobre contribuição do exercício aeróbio, de força e concorrente para a HPE, bem como apresenta possíveis mecanismos fisiológicos envolvidos. A ocorrência de HPE após diferentes tipos de exercício parece ser bem-aceita, tanto em indivíduos normotensos, quanto hipertensos. Contudo, a dose ótima de exercício aeróbio (ex.: relação entre intensidade, duração, modo de exercício e forma de execução) e de força (ex.: relação entre intensidade, volume e massa muscular envolvida) para maximizá-la permanece incerta. Dúvidas também persistem em relação aos diversos mecanismos fisiológicos envolvidos na HPE, que parecem ser diferentes no exercício aeróbio e de força. Destacam-se os mecanismos

Revista HUPE, Rio de Janeiro, 2013;12(4):99-110 doi:10.12957/rhupe.2013.8717 centrais e locais associados, respectivamente, à diminuição do débito cardíaco (DC) e resistência vascular periférica (RVP). Nesse sentido, os mecanismos envolvidos na HPE após o exercício aeróbio associar-se-iam tanto a fatores centrais (ex.: diminuição da atividade nervosa simpática), quanto a fatores periféricos (ex.: vasodilatação sustentada pela liberação de óxido nítrico, prostaglandinas e receptores da histamina). Na força, a HPE parece relacionarse, principalmente, com a diminuição do DC e a queda do volume sistólico, em resposta à menor perfusão miocárdica ocasionada pela maior compressão sequencial dos vasos.

Descritores: Pressão arterial; Fisiologia cardiovascular; Exercício; Promoção da saúde.

\section{Abstract}

Evidence indicates that chronic reduction in blood pressure (BP) due to physical exercise depends on the acute decrease in BP after a training bout, phenomenon referred as post- 
exercise hypotension (PEH). This study reviews research on PEH after acute aerobic, strength and concurrent exercise and its potential underlying mechanisms. The $\mathrm{PEH}$ has been observed following different exercise modalities in both normotensive and hypertensive subjects. However, the optimal dose of aerobic exercise [i.e. relationship between intensity, duration, mode and form of execution] and strength training [i.e. relationship between intensity, volume and muscle mass involved] to maximize its occurrence remains unclear. Questions also persist with regard to the physiological pathways of $\mathrm{PEH}$, which seem to be different in strength and aerobic exercise. Central and local mechanisms associated to decreased cardiac output (CO) and peripheral vascular resistance (PVR) are frequently mentioned in the literature. Mechanisms underlying $\mathrm{PEH}$ after aerobic exercise seem to include both central [i.e. decreased activity sympathetic nerve] and peripheral factors [i.e. vasodilation sustained by nitric oxidereleasing, prostaglandins and histamine receptors]. On the other hand, after strength or concurrent training, the $\mathrm{PEH}$ seems to be mostly related to a decrease in $\mathrm{CO}$ and systolic volume, in response to a decrease in myocardial perfusion during exercise due to compression of vessels by sequential muscle contraction.

Keywords: Blood pressure; Cardiovascular physiology; Exercise; Health promotion.

\section{Introdução}

A hipertensão arterial (HA) é um problema grave em saúde pública, associando-se a um maior risco para o desenvolvimento de diversas outras doenças, como disfunção renal e doenças cardíacas. ${ }^{1}$ No ano 2000 , mais de um quarto da população mundial era hipertensa $(26,4 \%$; 972 milhões) e, em 2025, esse número poderá atingir aproximadamente $29,2 \%$ ou 1,56 bilhão de pessoas no mundo. ${ }^{2}$ Se por um lado a HA é apontada como uma morbidade preocupante, por outro a prática regular de exercício físico tem sido frequentemente recomendada como uma conduta fundamental na prevenção ou no tratamento não farmacológico dessa doença. ${ }^{3}$

Diversos estudos demonstraram que uma única sessão de exercício aeróbio, ${ }^{4-11}$ de força ${ }^{12-17}$ ou concorrente (aeróbio e força) ${ }^{18-21}$ seria capaz de reduzir a pressão arterial (PA) por algumas horas. Esse fenômeno, conhecido na literatura como hipotensão pós-exercício (HPE), consiste em níveis pressóricos observados no período de recuperação inferiores àqueles aferidos na situação pré-treino ${ }^{22}$ ou mesmo àqueles verificados em um dia-controle sem realizar exercícios. ${ }^{3}$ Por outro lado, há evidências de que reduções crônicas da PA, em virtude da prática continuada de programas de exercício, devem-se, em grande medida, ao somatório dos efeitos hipotensivos agudos das sessões de treinamento. ${ }^{23}$ Trata-se, portanto, de um efeito desejável, justificando o interesse na investigação sobre possíveis maneiras de se maximizálo. Porém, dúvidas persistem a respeito da influência das variáveis do treinamento aeróbio e de força sobre a magnitude e/ou duração dessa resposta, bem como, no tocante aos mecanismos fisiológicos associados à HPE.

Desse modo, a presente revisão apresenta estudos que examinaram a contribuição do exercício aeróbio, de força e concorrente para a HPE. Adicionalmente, buscou-se analisar possíveis mecanismos fisiológicos subjacentes ao efeito, em cada tipo de exercício. Para tanto, o texto foi organizado nas seguintes seções: a) respostas ao treinamento aeróbio; b) respostas ao treinamento de força; $\mathrm{c}$ ) respostas ao treinamento concorrente; d) principais mecanismos fisiológicos associados à HPE.

\section{Respostas ao treinamento aeróbio}

No tocante ao efeito hipotensor pósexercício, vários aspectos relacionados à prescrição do treinamento aeróbio permanecem obscuros, como a influência da intensidade, duração, modo de exercício ou forma de execução (sessões contínuas ou fracionadas). 
Por exemplo, os resultados dos estudos em relação à magnitude e duração dos efeitos hipotensivos em função da intensidade são conflitantes. $^{24}$

As evidências disponíveis sustentam que sujeitos normotensos e hipertensos apresentariam redução significativa da PA após a realização de uma simples sessão de exercício aeróbio, por um período expressivo nas primeiras 24 horas, com diminuição de maior magnitude e duração nas pessoas hipertensas. ${ }^{3}$ Por exemplo, dados extraídos de uma revisão feita por Kenney e Seals ${ }^{25}$ indicam que, em jovens saudáveis, o treinamento aeróbio realizado com intensidade moderada e duração entre $30 \mathrm{e}$ 60 minutos promoveriam, após sua execução, declínios na pressão arterial sistólica (PAS) e pressão arterial diastólica (PAD), da ordem de $10 \mathrm{mmHg}$ e $5 \mathrm{mmHg}$, por até quatro horas. Todavia, esses efeitos são mais pronunciados em pessoas hipertensas, alcançando cifras de $20 \mathrm{mmHg}$ para PAS e de $9 \mathrm{mmHg}$ para PAD, permanecendo por até 13 horas.

Alguns estudos verificaram que exercícios aeróbios de intensidade vigorosa produziram maior magnitude $e^{4,6,26,27}$ e duração ${ }^{4,27} \mathrm{da}$ hipotensão, quando comparados a exercícios de intensidade moderada. Todavia, outras investigações não encontraram diferenças na magnitude e duração da HPE que pudessem ser atribuídas à intensidade do exercício em sujeitos normotensos ${ }^{5}$ e hipertensos. ${ }^{11}$ Em relação à duração, a hipotensão tem sido observada após exercícios de curta $(10 \mathrm{~min})^{28}$ e longa duração (170 min). ${ }^{29}$ Estudos que comparam a HPE após exercícios com diferentes durações, em normotensos e hipertensos, demonstraram que sessões com maior duração potencializariam tanto a magnitude quanto a duração da hipotensão. ${ }^{30,31}$ Entretanto, outros estudos não apontaram impacto da duração da sessão de exercício na magnitude ou duração da hipotensão, também em sujeitos normotensos ${ }^{28}$ e hipertensos. $^{32}$

Nota-se, portanto, não ser possível afirmar de forma definitiva se a duração de uma sessão de exercício aeróbio modula a magnitude e a duração da hipotensão. Por outro lado, dados recentes mostram que a relação entre intensidade e duração parece ser mais determinante na produção da HPE em normotensos, do que a ação isolada dessas variáveis. ${ }^{6}$ Em outras palavras, uma única sessão de exercício aeróbio com menor intensidade e longa duração poderia ocasionar os mesmos resultados de hipotensão que uma sessão de alta intensidade e curta duração. Infelizmente, com exceção do trabalho de Jones e colaboradores, ${ }^{6}$ nenhum estudo prévio investigou a resposta hipotensora ao exercício aeróbio a partir de sessões isocalóricas de exercício com diferentes intensidades e durações.

Destaca-se também a influência do modo de exercício ou da massa corporal total envolvida num determinado exercício sobre as respostas hipotensoras. São raros os estudos que buscaram comparar diretamente o efeito de diferentes tipos de exercício sobre o comportamento da HPE. MacDonald e colaboradores, ${ }^{28}$ por exemplo, concluíram que 30 minutos de exercício de intensidade vigorosa $\left(65-70 \% \mathrm{VO}_{2}\right.$ pico) realizado com maior massa muscular (ergômetro de perna) poderiam induzir HPE de maior duração (mas não magnitude) que exercício realizado com menor massa muscular (ergômetro de braço). No que tange à forma de execução, Jones e colaboradores ${ }^{7}$ compararam as respostas de PA após sessões contínuas (30 min) e fracionadas ( 3 sessões de 10 min com intervalo de 10 min de repouso) em cicloergômetro, com intensidade de $70 \% \mathrm{VO}_{2}$ pico em sujeitos normotensos e fisicamente ativos. A sessão fracionada proporcionou maior hipotensão do que a sessão contínua $(\mathrm{P}<0,05)$. Achados semelhantes foram verificados por Park e colaboradore ${ }^{10}$ em indivíduos pré-hipertensos que executaram exercícios de caminhada, de forma fracionada (3-4 sessões de $10 \mathrm{~min}$ com intervalo de recuperação de $50 \mathrm{~min}$ ) e contínua (30-40 min), com intensidade de $50 \% \mathrm{VO}_{2}$ pico.

Verificou-se, igualmente, que uma mudança na modulação simpática (decréscimo no tônus 
simpático e aumento no tônus parassimpático) associava-se estreitamente com a redução da PA após cada sessão de exercício. ${ }^{9,10} \mathrm{Na}$ verdade, isso se faz importante quando se lida com indivíduos hipertensos, uma vez que apresentam uma ativação exacerbada do sistema autônomo simpático em repouso. De todo modo, a magnitude da HPE parece estar estreitamente relacionada a uma maior PA em repouso, ${ }^{33}$ havendo mais chance de observá-la em indivíduos com PA elevada do que naqueles em que seus valores são naturalmente baixos.

\section{Respostas cardiovasculares agudas ao treinamento de força}

A prática do treinamento de força está consolidada na literatura por promover ganhos funcionais relacionados à prevenção de problemas osteomioarticulares e melhoria do condicionamento físico, sendo, inclusive, uma atividade fundamental em programas de reabilitação cardiovascular. ${ }^{34}$ As adaptações neuromusculares e hemodinâmicas decorrentes dessa prática contribuem para um menor esforço relativo durante atividades submáximas e, consequentemente, para um menor estresse cardiovascular, quantificado principalmente com base em respostas pressóricas. ${ }^{34}$ Todavia, a relação entre treinamento da força e HPE ainda não é clara, principalmente no que diz respeito a como intensidade (carga) e volume (massa muscular, número de séries e repetições) de treinamento interagem para provocá-la.

\section{Volume do treinamento de força e HPE}

O efeito do volume do treinamento de força sobre a HPE tem sido investigado em relação ao número de séries, repetições e quantidade de exercícios aplicados. Scher e colaboradores ${ }^{15}$ investigaram as respostas cardiovasculares durante 60 minutos, após 20 minutos e 40 minutos de treinamento de força em circuito, com intensidade de $40 \%$ de uma repetição máxima (RM) em 16 idosos hipertensos. Houve HPE após ambos os protocolos, embora somente a sessão com maior volume tenha reduzido a PAS após 24 horas. ${ }^{15}$ Apesar da baixa intensidade, o treinamento de força em circuito favorece um maior número de repetições e maior tempo de tensão muscular, o que pode contribuir para uma maior resposta cardiovascular em virtude de maior estimulação nervosa muscular aferente, mediada por mecanismos associados aos controles mecanorreflexo e metaborreflexo.

Outra consequência do treinamento é a compressão sequencial dos vasos sanguíneos, que promove aumento na resistência vascular periférica (RVP) e tende a diminuir a perfusão miocárdica. ${ }^{35}$ Considerando o efeito cumulativo das respostas pressóricas ao longo das séries, o fluxo sanguíneo do antebraço (FSA), após aplicação de séries múltiplas, tem sido alvo de investigações, pois possibilita uma análise da capacidade vasodilatadora pós-esforço, por sua vez relacionada à HPE. Nesse sentido, esse estudo de nosso grupo ${ }^{36}$ analisou tais respostas durante 60 minutos pós-exercício em nove homens que executaram 10 séries de 15 repetições a $90 \%$ da carga para 15 RM na extensão bilateral do joelho. A PA, FSA e hiperemia reativa foram medidas após 10 minutos e 60 minutos de repouso. Observou-se declínio da PA aos 10 minutos de repouso. Essa redução deu-se paralelamente a um declínio do FSA. Nenhum efeito foi observado nas situações-controle e exercício para a hiperemia reativa ou para a reatividade vascular (obtida pela diferença entre FSA imediatamente após a hiperemia reativa e em repouso). Concluiu-se que a HPE aos exercícios de força poderia ser menos dependente de respostas vasodilatadoras e aumento do fluxo sanguíneo periférico, do que o usualmente observado em treinamento aeróbio.

Intensidade do treinamento de força e HPE

Estudos prévios revelaram que uma maior carga mobilizada durante exercícios 
de força exacerbam as respostas pressóricas, provavelmente em virtude de uma maior aferência simpática e menor volume sistólico (VS), como consequência da maior RVP. ${ }^{35}$ Apesar disso, os estudos ainda são contraditórios no que tange ao efeito da intensidade do treinamento de força sobre possíveis respostas hipotensivas.

Alguns estudos de nosso grupo investigaram o papel relativo de intensidade e volume em sessões de treinamento da força. ${ }^{14,16}$ Polito e colaboradores ${ }^{14}$ avaliaram a influência de diferentes intensidades em protocolos pareados pelo volume (produto entre séries, repetições e carga) em 16 voluntários que realizaram dois protocolos em circuito: a) três séries com $6 \mathrm{RM}$ (SEQ6); b) 3 séries de 12 repetições com carga correspondente a 50\% 6 RM (SEQ12). Os efeitos dos dois protocolos foram similares sobre a PAS, que permaneceu reduzida após cerca de uma hora, enquanto pouco ou nenhum efeito hipotensivo foi observado para a PAD. Simão e colaboradores ${ }^{16}$ aplicaram protocolo similar a 14 homens que realizaram três séries de 6 RM realizadas em cinco (G1) e em seis exercícios (G2). Além disso, também foi aplicado um circuito com 12 repetições fixas a 50\% de 6 $\mathrm{RM}$, para ambos os grupos. Os resultados praticamente reproduziram os achados de Polito e colaboradores. ${ }^{14}$

Em contrapartida, Simões e colaboradores ${ }^{17}$ observaram o efeito do treinamento de maior intensidade sobre a duração e magnitude da HPE. Vinte indivíduos, dez diabéticos do tipo 2 e dez não diabéticos, foram submetidos a dois protocolos em circuito (16 e 30 repetições a $43 \%$ e $23 \%$ de $1 \mathrm{RM}$, respectivamente). Os resultados revelaram efeito hipotensivo para a PAS e pressão arterial média (MAP) decorrente apenas da sessão mais intensa (43\% 1 RM), com redução mais prolongada da PAS em não diabéticos (120 min vs. $90 \mathrm{~min}$ ), além de uma menor magnitude da HPE no grupo diabético tipo 2. Uma possível disfunção endotelial no grupo diabético pode ter implicado em menor complacência arterial, maior RVP e consequente acúmulo de metabólitos resultantes da atividade, favorecendo o metaborreflexo e uma menor HPE.

As relações entre intensidade e volume ainda não estão claras, portanto. Contudo, embora não se tenham encontrado estudos observando esses mecanismos, pode-se especular que, talvez, o ponto-chave para explicar a HPE após exercícios de força esteja relacionado à definição dos papéis da intensidade ou volume de treinamento para o aumento de estímulos aferentes de natureza central (comando central) e periférica (ergorreflexo), resultando em um deslocamento (reset) do ponto de atuação barorreflexa em relação ao repouso. ${ }^{37}$

\section{Massa muscular recrutada e HPE}

De acordo com a teoria do reflexo pressórico do exercício (ou ergorreflexo), uma maior aferência muscular decorrente da excitação de ergorreceptores (mecanorreceptores e metaborreceptores) pode resultar em respostas hemodinâmicas mais exacerbadas. ${ }^{38}$ Pessoas com problemas cardiovasculares, a exemplo dos hipertensos, podem apresentar disfunções em reflexos cardiovasculares associados ao recrutamento de fibras musculares, a exemplo do ergorreflexo. Dessa forma, o efeito da magnitude da massa muscular recrutada (também um componente do volume) sobre as respostas cardiovasculares pós-exercício merece investigação.

Tradicionalmente, respostas cardiovasculares mais elevadas têm sido associadas a um maior recrutamento de unidades motoras (drive motor), resultante de uma maior descarga aferente dos centros cerebrais superiores (comando central) e do ergorreflexo. ${ }^{35,38}$ Poucos estudos investigaram o efeito de diferentes magnitudes de recrutamento muscular sobre a HPE. Polito e Farinatti ${ }^{12}$ avaliaram esse efeito ao longo de 60 minutos, a partir da comparação entre os exercícios de rosca, bíceps e extensão dos joelhos aplicados com 6 e 10 séries de 12 RM, em 24 homens treinados. A HPE foi observada apenas no exercício destinado ao maior grupo muscular (quadríceps), quando realizado com maior 
volume de séries múltiplas. Embora não tenha comparado a HPE decorrente de diferentes magnitudes de massa muscular, resultados similares foram encontrados por Mediano e colaboradores ${ }^{39}$ após os exercícios supino reto, leg press horizontal, remada alta e rosca tríceps, em relação às respostas cardiovasculares pósesforço. O estudo foi aplicado com uma e três séries em hipertensos controlados, revelando maior duração de efeito hipotensivo para PAS e PAD após três séries. Um estresse de cisalhamento (shear stress) elevado, provavelmente observado em grupos musculares de maior volume, sugere uma maior liberação de substâncias vasodilatadoras, a exemplo do óxido nítrico $\left(\mathrm{NO}_{2}\right)$ e das prostaglandinas, e consequente redução da RVP, que ajudaria a provocar efeito hipotensivo.

Alguns estudos têm avaliado o efeito da magnitude da massa muscular recrutada sobre tais respostas, aplicando-se padrões diferentes de execução para um mesmo exercício. Por exemplo, um mesmo grupamento muscular pode ser observado a partir de diferentes níveis de recrutamento muscular, assumindo-se que o exercício bilateral recrute o dobro de massa muscular do unilateral. Polito e colaboradores ${ }^{13}$ aplicaram o exercício de extensão uni e bilateral dos joelhos em 18 indivíduos (seis homens), avaliando as respostas de PAS, PAD, frequência cardíaca (FC) e duplo-produto (DP) durante 20 minutos de recuperação. Não houve diferença entre os protocolos. O período mais longo de exposição ao estímulo durante a execução unilateral, que resulta em maior tempo de tensão muscular, talvez tenha sido suficiente para gerar uma descarga adrenérgica de maior duração, mas de magnitude equivalente à apresentada no exercício com maior massa muscular.

\section{Respostas cardiovasculares agudas ao treinamento concorrente}

O treinamento de força tem sido recomendado como complementar ao aeróbio, com a finalidade de reforçar o tratamento, controle ou mesmo a prevenção de doença cardiovascular. ${ }^{3}$ Contudo, a influência da associação dessas formas de treinamento em uma mesma sessão (denominada treinamento concorrente), nas respostas cardiovasculares pós-exercício ainda são pouco conhecidas. $\mathrm{Na}$ verdade, apenas quatro estudos investigaram os efeitos do treinamento concorrente sobre as respostas cardiovasculares pós-exercício. ${ }^{18-21}$

A principal hipótese acerca do treinamento concorrente consistiria em um efeito hipotensor aditivo, gerado pela associação de ambas as atividades. No estudo de Teixeira e colaboradores, ${ }^{21} 20$ indivíduos realizaram treinamento concorrente composto por exercício aeróbio em cicloergômetro com intensidade de $75 \% \mathrm{VO}_{2}$ pico, seguido por seis exercícios de força com três séries de 20 repetições cada, com $50 \%$ de 1 RM. A sessão concorrente durou 60 minutos, o dobro de cada sessão isolada. As variáveis cardiovasculares foram avaliadas ao longo de 120 minutos de recuperação, sendo encontrada maior redução da PAS após a atividade aeróbia e concorrente, e redução mais prolongada da PAD somente após a aeróbia. Verificou-se redução do DC e VS em todos os exercícios, além de aumento da RVP após os exercícios de força e concorrente, com respostas mais elevadas de FC após o concorrente. Embora existente, o efeito hipotensivo não foi potencializado com o treinamento concorrente, talvez devido à ordem dos exercícios aplicados, o que favoreceu o componente estático da força, resultando em maior magnitude da RVP, não compensada por um aumento do DC.

Em decorrência da maior RVP após o exercício de força, é plausível que posicioná-lo antes do aeróbio em uma sessão de treinamento concorrente evite comprometer o efeito hipotensivo. Em estudo de nosso grupo, 21 homens foram submetidos a um treinamento concorrente, composto por duas séries de seis a oito repetições com $80 \% 1 \mathrm{RM}$ em oito exercícios, seguidos por 30 minutos de exercício aeróbio em cicloergômetro a $65 \% \mathrm{VO}_{2}$ pico. ${ }^{19}$ 
Os sujeitos também realizaram sessões de treinamento aeróbio e força, com os mesmos componentes, mas duração prolongada (3 séries para o treinamento da força e 60 minutos de cicloergômetro para o treinamento aeróbio), a fim de parear as sessões pela duração total (aproximadamente 60 minutos). A magnitude da PAS e PAD ao longo dos 120 minutos de recuperação foi similar nos três tipos de treinamento. No entanto, os exercícios aeróbio e concorrente provocaram HPE de maior duração para PAS, enquanto somente o aeróbio induziu hipotensão mais prolongada para a PAD. O efeito hipotensivo do treinamento concorrente foi, sem dúvida, superior ao do treinamento de força isolado. É possível que, nesta ordem de execução, a menor complacência observada agudamente após treinamento de força seja suplantada por uma maior liberação de substâncias vasodilatadoras após o treinamento aeróbio.

Ruiz e colaboradores ${ }^{20}$ compararam os efeitos de sessões isoladas e concorrentes sobre as respostas cardiovasculares pós-exercício em 11 homens submetidos a uma sessão aeróbia composta por 40 minutos em cicloergômetro com $60-70 \%$ do $\mathrm{VO}_{2}$ de reserva, assim como a uma sessão de força com três séries de 12 RM em oito exercícios destinados aos principais grupos musculares. Além de o treinamento concorrente ter sido realizado com maior volume, a atividade aeróbia foi precedida à de força, o que pode implicar em efeitos hipotensivos de menor magnitude. ${ }^{19}$ Os resultados indicaram HPE (PAS e MAP) após todas as sessões, mas valores significativos para a redução da PAS também foram observados após 30 minutos da finalização do exercício aeróbio. De forma semelhante, o período pós-atividade aeróbia demonstrou não somente uma FC elevada por um menor período de tempo (até 15 minutos), como também uma menor magnitude de reentrada vagal em comparação com as demais sessões.

Diante da maior eficácia do treinamento aeróbio isolado em promover HPE, a manipulação da intensidade do exercício aeróbio aplicado no treinamento concorrente pode influenciar nas possíveis respostas hipotensivas. Estudo de nosso grupo comparou as intensidades de $50 \%, 65 \%$ e $80 \%$ do $\mathrm{VO}_{2}$ pico em exercício aeróbio realizado após exercícios de força em sessões concorrentes. ${ }^{18}$ Vinte e um homens realizaram três sessões compostas por seis exercícios de força (2 séries de 6 a 8 repetições a $80 \%$ de $1 \mathrm{RM}$ ), sempre seguidos por 30 minutos em cicloergômetro. Foram encontradas diferenças em relação à duração da HPE para a PAS, aproximadamente uma hora mais prolongada no protocolo com maior intensidade, enquanto a PAD teve afetadas tanto sua magnitude (ligeiramente superior em 65\% e $80 \%$ do que em $50 \% \mathrm{VO}_{2}$ pico), quanto sua duração, maior após o protocolo mais intenso. Do ponto de vista prático, recomendar-se-ia uma intensidade maior que $65 \%$ do $\mathrm{VO}_{2}$ pico no exercício aeróbio da sessão concorrente, para obtenção de HPE mais expressiva.

\section{Principais mecanismos fisiológicos associados à HPE}

\section{Mecanismo central da HPE}

As principais áreas cerebrais responsáveis pelo controle cardiovascular incluem: hipotálamo lateral, medula caudal ventrolateral (MCVL), medula rostral ventrolateral (MRVL) e núcleo do trato solitário (NTS). ${ }^{40}$ O NTS é a primeira região central na qual ocorrem as interações neuronais aferentes do controle neural cardiovascular. Portanto, ele pode ser considerado como umas das principais estruturas responsáveis por essa regulação. $\mathrm{O}$ balanço final dessas interações resultará em um reajuste do funcionamento do barorreflexo, com atividade nervosa simpática aumentada ou diminuída sobre vasos e coração. Em última instância, a PA é aumentada ou diminuída em função de maior ou menor RVP ou DC. ${ }^{41}$

A fim de esclarecer os mecanismos envolvidos na HPE, alguns modelos foram propostos. Potts ${ }^{42}$ sugere que a FC e a atividade 
nervosa simpática sejam controladas por motoneurônios vagais cardíacos e neurônios inibitórios GABA, que inibem, por sua vez, neurônios pré-motores simpáticos na MRVL. Essa atividade central seria modulada a partir da magnitude da eferência excitatória de neurônios barossensitivos do NTS, controlados por intermédio do arco aórtico e seio carotídeo. Desse modo, sugere-se uma neurotransmissão inibitória no NTS, que repercute sobre a atividade barorreflexa mediante um controle de feedback negativo. No entanto, caso não ocorra inibição do NTS, o consequente aumento da atividade barorreflexa pode limitar a magnitude da retirada vagal e respostas simpatoexcitatórias decorrentes do exercício.

Outro modelo, proposto por Chen e Bonham, ${ }^{41}$ foca a aferência muscular ergorreflexa no NTS como ponto-chave para a resposta eferente simpática dos centros de controle cardiovascular, sobre a modulação cardíaca a vasomotora. Durante o exercício, o NTS receberia estímulos aferentes musculares em resposta à atividade muscular aumentada, estimulando a liberação da substância $\mathrm{P}$, se ligaria a interneurônios barossensitivos de segunda ordem, a partir de receptores GABAérgicos NK-1, que por sua vez liberam GABA, um neurotransmissor inibitório. Os neurônios barossensitivos de segunda ordem transmitem a informação aferente barossensitiva aos neurônios da MVLC, que passa essa informação, com uma menor inibição simpática proveniente da atividade GABAérgica, para os neurônios da MVLR. Os neurônios da MVLR (excitatórios) aumentam o disparo simpático vasoconstrictor e é observado um reajuste do ponto central de operação barorreflexa para cima.

Como resultado de uma atividade muscular aumentada, os receptores NK-1 são internalizados nos interneurônios GABA após o exercício. Com isso, os receptores NK-1 estariam menos disponíveis para se ligarem à substância $\mathrm{P}$ após o exercício e a atividade GABAérgica é diminuída. A partir disso, a atividade dos neurônios da MVLC aumenta em virtude de uma menor inibição pela interação GABA-P. Em consequência, a atividade excitatória dos neurônios da MVLR é diminuída, resultando em reajuste do barorreflexo para baixo, o que diminui a eferência simpática pós-exercício. ${ }^{41}$

A atividade nervosa simpática diminuída após o exercício ocasiona diminuição da RVP e, em alguns momentos, do DC. ${ }^{22}$ Uma vez que a PA é composta pelo produto do DC pela RVP, é possível entender parte dos mecanismos envolvidos na HPE. No entanto, a questão da intensidade e volume continua indefinida. Fica claro que a atividade muscular aumentada durante o exercício (liberando substância P) é um aspecto-chave para entender como a HPE se manifesta - estudos futuros deverão investigar se esse aumento na atividade muscular e, portanto, na liberação da substância $\mathrm{P}$, estaria mais ligado à intensidade (cargas) dos diferentes tipos de exercício ou a aspectos mais relacionados ao seu volume (massa muscular, gasto calórico, etc.).

\section{Mecanismo periférico de HPE}

O mecanismo periférico da HPE está intimamente relacionado com a vasodilatação local, como reflexo da atividade metabólica durante o exercício. Em decorrência do esforço, o DC aumenta a fim de compensar o aumento da RVP dos músculos inativos e garantir a redistribuição adequada do fluxo sanguíneo para os órgãos vitais. Durante a recuperação, o fenômeno da vasodilatação pode ser classificado em hiperemia (imediatamente após o exercício) e em vasodilatação sustentada pós-exercício. ${ }^{43}$ Sabe-se que a duração e a magnitude da hiperemia dependem de um estímulo suficiente para ativação dos músculos esqueléticos.

Em situação de exercício, uma série de mecanismos metabólicos e mecânicos podem influenciar a magnitude da vasodilatação local pós-exercício. Substâncias vasodilatadoras locais que são liberadas durante o exercício, podem ter contribuição potencial para a vasodilatação prolongada pós-exercício. ${ }^{22} \mathrm{~A}$ partir do desequilíbrio entre suprimento e demanda de oxigênio, a adenosina trifosfato 
(ATP) também exerce efeito vasodilatador, em consequência da redução da pressão parcial de $\mathrm{O}_{2}$ local. Esse efeito pode ocorrer de forma direta, com base na ativação de receptores purinérgicos $\mathrm{P} 2$, o que estimula a produção de NO e prostaglandinas; mas também de forma indireta, mediante a degradação das ATPs em adenosinas, seguida da ativação de seus respectivos receptores purinérgicos $\mathrm{P} 1$. Embora não seja um autêntico vasodilatador, uma concentração elevada de íons potássio $(\mathrm{K}+)$ (entre 4 e $20 \mathrm{mM}$ ) pode apresentar ação vasodilatadora, o que justifica a sua atividade associada à intensidade do exercício e a mudanças na condutância vascular. ${ }^{43}$

$\mathrm{O} \mathrm{NO}_{2}$ parece exercer um efeito importante sobre essa vasodilatação durante e após o exercício. Evidências sugerem que, em ratos, o óxido nítrico contribui para a vasodilatação sustentada pós-exercício, por meio de uma diminuição da sensibilidade dos receptores a-adrenérgicos. ${ }^{44} \mathrm{~A}$ vasodilatação promovida pela hiperemia atua em consonância com a função endotelial, cuja disfunção pode minimizar a liberação de metabólitos vasodilatadores decorrentes de um aumento do fluxo sanguíneo e do estresse de cisalhamento. A liberação de $\mathrm{NO}_{2}$, por exemplo, pode ser comprometida em virtude do polimorfismo genético da enzima eNOS (óxido nítrico sintase endotelial). Nesse sentido, um indivíduo acometido por esse polimorfismo produzirá menos $\mathrm{NO}_{2}$ e, consequentemente, poderá ser menos responsivo ao efeito do exercício sobre a PA. ${ }^{45}$ Entretanto, em humanos parece ocorrer um menor controle do tônus vascular mediado pelo $\mathrm{NO}_{2}$ em situação de recuperação ao exercício, ${ }^{22}$ mesmo com a sensibilidade dos receptores $\alpha$-adrenérgicos inalterada. ${ }^{46}$

Talvez, uma maior condutância vascular esperada durante a HPE (ao menos no exercício aeróbio) também possa ser atribuída à presença de prostaglandinas. No entanto, mesmo com a inibição da enzima cicloxigenase, que resulta na produção de prostaglandinas, não está evidenciado o efeito dessas substâncias sobre o aumento da condutância vascular sistêmica, pelo menos em indivíduos normotensos submetidos a uma hora de exercício aeróbio a $60 \% \mathrm{VO}_{2}$ pico. ${ }^{47}$ Estudos recentes do grupo do Halliwill têm mostrado que a vasodilatação local sustentada pós-exercício é dependente da ativação dos receptores da histamina $\mathrm{H} 1 \mathrm{e} \mathrm{H} 2$. A administração combinada de antagonistas do H1 e $\mathrm{H} 2$ pós-exercício reduziria a vasodilatação em $80 \%$ e a HPE em 65\%, após 60 min de exercício em cicloergômetro em intensidade moderada em indivíduos sedentários, fisicamente ativos e atletas. Além disso, a vasodilatação local e HPE foram inibidas quando administrados antagonistas dos receptores $\mathrm{H} 1$ e H2, após 60 minutos de exercício com intensidade moderada em extensão dinâmica unilateral de joelho. ${ }^{48}$

Dentre os fatores relacionados com a vasodilatação periférica pós-exercício até aqui mencionados, a via histaminérgica parece ser o mecanismo provocador da HPE mais importante em indivíduos normotensos e recreacionalmente ativos. Barrett-O'Keefe e colaboradores $^{49}$ investigaram até que ponto a vasodilatação seguida de exercício aeróbio unilateral de perna ( $60 \%$ da potência de pico), bem como de exercício de resistência de extensão de joelho ( $50 \%$ do pico de torque), era sustentada e mediada pelos receptores de histamina. Na situação-controle (sem bloqueio dos receptores de histamina), o exercício aeróbio aumentou a condutância vascular na perna exercitada em $27,2 \pm 8,4 \%(\mathrm{P}<0,05)$ em comparação aos valores pré-exercício, mas não na situação de bloqueio $(\Delta=3,7 \pm 5,1 \% ; \mathrm{P}=0,8)$. Os autores destacaram, ainda, que os efeitos do bloqueio sobre a condutância vascular foram restritos somente à perna exercitada.

Em relação ao exercício resistido, não houve nenhuma mudança na condutância vascular durante os 60 minutos pós-exercício $(\Delta=-$ $4,3 \pm 4,7 \% ; \mathrm{P}=0,7)$ e esta não foi afetada pelo bloqueio $(\mathrm{P}=0,7) .{ }^{49}$ Esses resultados indicam que os receptores de histamina são ativados, especialmente, após exercício aeróbio. Além disso, é possível afirmar que fatores locais 
associados ao exercício aeróbio, mas não fatores sistêmicos ou associados à força muscular elevada, seriam os responsáveis pela ativação dos receptores de histamina no músculo previamente exercitado.

\section{Conclusão}

Apesar de a ocorrência da HPE estar bem-descrita na literatura em populações com diferentes estados clínicos, vários aspectos permanecem obscuros. Destaca-se, nesse sentido, como combinar as variáveis de prescrição do treinamento aeróbio, de força e concorrente para otimizar a magnitude e duração HPE e, por conseguinte, o efeito crônico do treinamento sobre a PA. Há um maior número de evidências indicando presença de HPE após treinamento aeróbio, quando comparado ao treinamento de força. No entanto, é possível que o treinamento concorrente permita a ocorrência do efeito hipotensivo pós-exercício, permitindo ganhos simultâneos na função neuromuscular e cardiorrespiratória.

Os mecanismos envolvidos na HPE ainda necessitam ser melhor esclarecidos, devido à influência multifatorial de aspectos genéticos, metabólicos e mecânicos. De fato, a maior parte dos estudos investigou o fenômeno em animais submetidos ao exercício aeróbio, o que limita a validade externa de outras formas de exercício aplicadas em seres humanos. Apesar disso, nota-se que a participação integrada entre as variáveis cardiovasculares reflete mecanismos subjacentes ao controle neural do sistema nervoso autônomo.

Em suma, após o exercício aeróbio, pode-se postular que os mecanismos da HPE envolvem tanto fatores centrais (ex.: diminuição do DC em resposta ao decréscimo da atividade nervosa simpática, que por sua vez relaciona-se com a diminuição da atividade excitatória dos neurônios da MVLR, resultando em reajuste do barorreflexo para baixo e na diminuição da eferência simpática pós-exercício), como periféricos (ex.: redução da RVP em resposta a vasodilatação local sustentada pela liberação de
$\mathrm{NO}_{2}$, prostaglandinas e receptores da histamina $\mathrm{H} 1 \mathrm{e} \mathrm{H} 2)$. Por outro lado, a HPE decorrente do treinamento de força, em grande parte, parece envolver mecanismos centrais, dentre os quais a diminuição do DC e VS. Esse declínio aconteceria em resposta ao decréscimo da perfusão miocárdica determinada pelo aumento da RVP, em virtude de maior compressão sequencial dos vasos sanguíneos (influenciada pelo tempo de tensão muscular). No treinamento concorrente, enfim, os mecanismos envolvidos na HPE seriam similares aos da força, apesar de muito poucos estudos sobre o tema terem sido publicados.

\section{Referências}

1. Go AS, Mozaffarian D, Roger VL, Benjamin EJ, Berry JD, Borden WB, et al. Heart disease and stroke statistics--2013 update: a report from the American Heart Association. Circulation. 2013 Jan 1;127(1):e6-e245. http:// dx.doi.org/10.1161/CIR.0b013e31828124ad

2. Kearney PM, Whelton M, Reynolds K, Muntner P, Whelton PK, He J. Global burden of hypertension: analysis of worldwide data. Lancet. 2005 Jan 15-21;365(9455):217-23. http:// dx.doi.org/10.1016/S0140-6736(05)17741-1

3. Pescatello LS, Franklin BA, Fagard R, Farquhar WB, Kelley GA, Ray CA. American College of Sports Medicine position stand. Exercise and hypertension. Med Sci Sports Exerc. 2004 Mar;36(3):533-53. http://dx.doi. org/00005768-200403000-00025 [pii].

4. Forjaz CL, Cardoso CG Jr, Rezk CC, Santaella DF, Tinucci T. Postexercise hypotension and hemodynamics: the role of exercise intensity. J Sports Med Phys Fitness. 2004 Mar;44(1):5462.

5. Forjaz CL, Matsudaira $Y$, Rodrigues FB, Nunes N, Negrao CE. Post-exercise changes in blood pressure, heart rate and rate pressure product at different exercise intensities in normotensive humans. Braz J Med Biol Res. 1998 Oct;31(10):1247-55. http:// dx.doi.org/10.1590/S0100-879X1998001000003

6. Jones H, George K, Edwards B, Atkinson $\mathrm{G}$. Is the magnitude of acute post-exercise hypotension mediated by exercise intensity or total work done? Eur J Appl Physiol. 2007 Dec;102(1):33-40. http://dx.doi.org/10.1007/ s00421-007-0562-0

7. Jones H, Taylor CE, Lewis NC, George K, Atkinson G. Post-exercise blood pressure reduction is greater following intermittent than continuous exercise and is influenced 
less by diurnal variation. Chronobiol Int. 2009 Feb;26(2):293-306. http://dx.doi. org/10.1080/07420520902739717

8. MacDonald JR, MacDougall JD, Hogben CD. The effects of exercising muscle mass on post exercise hypotension. J Hum Hypertens. 2000 May;14(5):317-20. http://dx.doi.org/10.1038/ sj.jhh.1000999

9. Park S, Rink L, Wallace J. Accumulation of physical activity: blood pressure reduction between 10-min walking sessions. J Hum Hypertens. 2008 Jul;22(7):475-82. http:// dx.doi.org/10.1038/jhh.2008.29

10. Park S, Rink LD, Wallace JP. Accumulation of physical activity leads to a greater blood pressure reduction than a single continuous session, in prehypertension. J Hypertens. 2006 Sep;24(9):1761-70. http://dx.doi. org/10.1097/01.hjh.0000242400.37967.54

11. Pescatello LS, Guidry MA, Blanchard BE, Kerr A, Taylor AL, Johnson AN, et al. Exercise intensity alters postexercise hypotension. J Hypertens. 2004 Oct;22(10):1881-8. http:// dx.doi.org/00004872-200410000-00009 [pii].

12.Polito MD, Farinatti PTV. The effects of muscle mass and number of sets during resistance exercise on postexercise hypotension. J Strength Cond Res. 2009 Nov;23(8):2351-7. http://dx.doi.org/10.1519/ JSC.0b013e3181bb71aa.

13. Polito MD, Rosa CC, Schardong P. Respostas cardiovasculares agudas na extensão do joelho realizada em diferentes formas de execução. Rev Bras Med Esporte. 2004 Jun;10(3):173-6. http://dx.doi.org/10.1590/ S1517-86922004000300006

14. Polito MD, Simão R, Senna GW, Farinatti PTV. Efeito hipotensivo do exercício de força realizado em intensidades diferentes e mesmo volume de trabalho. Rev Bras Med Esporte. 2003;9(2):69-73. http://dx.doi. org/10.1590/S1517-86922003000200003

15. Scher LM, Ferriolli E, Moriguti JC, Scher $\mathrm{R}$, Lima NK. The effect of different volumes of acute resistance exercise on elderly individuals with treated hypertension. J Strength Cond Res. 2011 Apr;25(4):1016-23. http://dx.doi.org/10.1519/ JSC.0b013e3181c70b4f

16. Simão R, Fleck SJ, Polito M, Monteiro W, Farinatti P. Effects of resistance training intensity, volume, and session format on the postexercise hypotensive response. J Strength Cond Res. 2005 Nov;19(4):853-8.

17. Simões GC, Moreira SR, Kushnic MR, Simões HG, Campbell CSG. Postresistance exercise blood pressure reduction is influenced by exercise intensity in type-2 diabetic and nondiabetic individuals. J Strength Cond Res. 2010 May;24(5):1277-84. http://dx.doi.
org/10.519/JSC.0b013e3181d67488

18. Keese F, Farinatti P, Pescatello L, Cunha FA, Monteiro WD. Aerobic exercise intensity influences hypotension following concurrent exercise sessions. Int J Sports Med. 2012 Feb;33(2):148-53. http://dx.doi. org/10.1055/s-0031-1291321

19. Keese F, Farinatti P, Pescatello L, Monteiro W. A comparison of the immediate effects of resistance, aerobic, and concurrent exercise on postexercise hypotension. J Strength Cond Res. 2011 May;25(5):1429-36. http://dx.doi. org/10.519/JSC.0b013e3181d6d968

20. Ruiz RJ, Simão R, Saccomani MG, Casonatto J, Alexander JL, Rhea M, et al. Isolated and combined effects of aerobic and strength exercise on post-exercise blood pressure and cardiac vagal reactivation in normotensive men. J Strength Cond Res. 2011 Mar;25(3):640-5. http://dx.doi.org/10.1519/ JSC.0b013e3181c1fcc7

21. Teixeira L, Ritti-Dias RM, Tinucci T, Mion Junior D, Forjaz CLM. Post-concurrent exercise hemodynamics and cardiac autonomic modulation. Eur J Appl Physiol. 2011 Sep;111(9):2069-78. http://dx.doi. org/10.1007/s00421-010-1811-1

22. Halliwill JR. Mechanisms and clinical implications of post-exercise hypotension in humans. Exerc Sport Sci Rev. 2001 Apr;29(2):65-70.

23. da Nobrega AC. The subacute effects of exercise: concept, characteristics, and clinical implications. Exerc Sport Sci Rev. 2005 Apr;33(2):84-7. http://dx.doi.org/00003677200504000-00005 [pii]

24. Casonatto J, Polito MD. Hipotensão pósexercício aeróbio: uma revisão sistemática. Rev Bras Med Esporte. 2009 MarApr;15(2)151-7.

25. Kenney MJ, Seals DR. Postexercise hypotension. Key features, mechanisms, and clinical significance. Hypertension. 1993 November 1, 1993;22(5):653-64. http://dx.doi. org/10.1161/01.hyp.22.5.653.

26. Alderman BL, Arent SM, Landers DM, Rogers TJ. Aerobic exercise intensity and time of stressor administration influence cardiovascular responses to psychological stress. Psychophysiology. 2007 Sep;44(5):75966. http://dx.doi.org/10.1111/j.14698986.2007.00548.x

27. Piepoli M, Isea JE, Pannarale G, Adamopoulos S, Sleight P, Coats AJ. Load dependence of changes in forearm and peripheral vascular resistance after acute leg exercise in man. J Physiol. 1994 Jul 15;478 (Pt 2):357-62.

28. MacDonald JR, MacDougall JD, Hogben CD. The effects of exercise duration on post- 
exercise hypotension. J Hum Hypertens. 2000 Feb;14(2):125-9.

29. Seals DR, Rogers MA, Hagberg JM, Yamamoto C, Cryer PE, Ehsani AA. Left ventricular dysfunction after prolonged strenuous exercise in healthy subjects. Am J Cardiol. 1988 Apr 15;61(11):875-9.

30. Bennett T, Wilcox RG, Macdonald IA. Post-exercise reduction of blood pressure in hypertensive men is not due to acute impairment of baroreflex function. Clin Sci (Lond). 1984 Jul;67(1):97-103.

31. Mach C, Foster C, Brice G, Mikat RP, Porcari JP. Effect of exercise duration on postexercise hypotension. J Cardiopulm Rehabil. 2005 Nov-Dec;25(6):366-9. http://dx.doi. org/00008483-200511000-00010 [pii]

32. Guidry MA, Blanchard BE, Thompson PD, Maresh CM, Seip RL, Taylor AL, et al. The influence of short and long duration on the blood pressure response to an acute bout of dynamic exercise. Am Heart J. 2006 Jun;151(6):1322 e5-12. http://dx.doi. org/10.1016/j.ahj.2006.03.010

33. Liu S, Goodman J, Nolan R, Lacombe S, Thomas SG. Blood pressure responses to acute and chronic exercise are related in prehypertension. Med Sci Sports Exerc. 2012 Sep;44(9):1644-52. http://dx.doi.org/10.1249/ MSS.0b013e31825408fb

34. Williams MA, Haskell WL, Ades PA, Amsterdam EA, Bittner V, Franklin BA, et al. Resistance exercise in individuals with and without cardiovascular disease: 2007 update: a scientific statement from the American Heart Association Council on Clinical Cardiology and Council on Nutrition, Physical Activity, and Metabolism. Circulation. 2007 Jul 31;116(5):572-84. http://dx.doi.org/10.1161/ CIRCULATIONAHA.107.185214

35. MacDougall JD, Tuxen D, Sale DG, Moroz JR, Sutton JR. Arterial blood pressure response to heavy resistance exercise. J Appl Physiol. 1985 Mar;58(3):785-90.

36. Polito MD, da Nóbrega AC, Farinatti P. Blood pressure and forearm blood flow after multiple sets of a resistive exercise for the lower limbs. Blood Press Monit. 2011 Aug;16(4):180-5. http://dx.doi.org/10.1097/ MBP.0b013e328348cac4

37. Fadel PJ, Raven PB. Human investigations into the arterial and cardiopulmonary baroreflexes during exercise. Exp Physiol. 2012 Jan;97(1):39-50. http://dx.doi.org/10.1113/ expphysiol.2011.057554

38. Mitchell JH, Kaufman MP, Iwamoto GA. The exercise pressor reflex: its cardiovascular effects, afferent mechanisms, and central pathways. Annu Rev Physiol. 1983;45:229-
42. http://dx.doi.org/10.1146/annurev. ph.45.030183.001305

39. Mediano MFF, Paravidino V, Simão R, Pontes FL, Polito MD. Comportamento subagudo da pressão arterial após o treinamento de força em hipertensos controlados. Rev Bras Med Esporte. 2005 Nov-Dec;11(6):337-40. http:// dx.doi.org/10.1590/S1517-86922005000600006

40. Yasui Y, Breder CD, Saper CB, Cechetto DF. Autonomic responses and efferent pathways from the insular cortex in the rat. J Comp Neurol. 1991 Jan 15;303(3):355-74. http:// dx.doi.org/10.1002/cne.903030303

41. Chen CY, Bonham AC. Postexercise hypotension: central mechanisms. Exerc Sport Sci Rev. 2010 Jul;38(3):122-7. http://dx.doi. org/10.1097/JES.0b013e3181e372b5

42. Potts JT. Inhibitory neurotransmission in the nucleus tractus solitarii: implications for baroreflex resetting during exercise. Exp Physiol 2006 Jan;91(1):59-72. http://dx.doi. org/10.1113/expphysiol.2005.032227

43. Laughlin $\mathrm{MH}$, Davis MJ, Secher $\mathrm{NH}$, van Lieshout JJ, Arce-Esquivel AA, Simmons GH, et al. Peripheral circulation. Compr Physiol. 2012 Jan; 2(1):321-447.

44. Patil RD, DiCarlo SE, Collins HL. Acute exercise enhances nitric oxide modulation of vascular response to phenylephrine. Am J Physiol. 1993 Oct;265(4 Pt 2):H1184-8.

45. Silva BM, Neves FJ, Rocha NG, Sales AR, Medeiros RF, Barbosa TC, et al. Endothelial nitric oxide gene haplotype reduces the effect of a single bout of exercise on the vascular reactivity in healthy subjects. Transl Res. 2013 Jan;161(1):15-25. http://dx.doi.org/10.1016/j. trsl.2012.05.004

46. Halliwill JR, Dinenno FA, Dietz NM. Alphaadrenergic vascular responsiveness during postexercise hypotension in humans. J Physiol. 2003 Jul 1;550(Pt 1):279-86. http:// dx.doi.org/10.1113/jphysiol.2003.042838

47. Lockwood JM, Pricher MP, Wilkins BW, Holowatz LA, Halliwill JR. Postexercise hypotension is not explained by a prostaglandin-dependent peripheral vasodilation. J Appl Physiol. 2005 Feb;98(2):447-53. http://dx.doi.org/10.1152/ japplphysiol.00787.2004

48. McCord JL, Halliwill JR. H1 and H2 receptors mediate postexercise hyperemia in sedentary and endurance exercise-trained men and women. J Appl Physiol. 2006 Dec;101(6):1693-701. http://dx.doi.org/10.1152/ japplphysiol.00441.2006

49. Barrett-O'Keefe Z, Kaplon RE, Halliwill JR. Sustained postexercise vasodilatation and histamine receptor activation following small muscle-mass exercise in humans. Exp Physiol. 2013 Jan;98(1):268-77. http://dx.doi. org/10.1113/expphysiol.2012.066605 


\section{Autores}

\section{Ada Fernanda P. S. Lima}

Laboratório de Atividade Física e Promoção da Saúde. Instituto de Educação Física e Desportos. Universidade do Estado do Rio de Janeiro. Rio de Janeiro, RJ, Brasil.

\section{Alexandre H. Okano}

Departamento de Educação Física. Centro de Ciências da Saúde. Universidade Federal do Rio Grande do Norte. Natal, RN, Brasil.

\section{Alice R. Sampaio}

Laboratório de Atividade Física e Promoção da Saúde. Instituto de Educação Física e Desportos. Universidade do Estado do Rio de Janeiro. Rio de Janeiro, RJ, Brasil.

\section{Ana Paula M. Guttierres}

Laboratório de Atividade Física e Promoção da Saúde. Instituto de Educação Física e Desportos. Universidade do Estado do Rio de Janeiro. Rio de Janeiro, RJ, Brasil.

\section{Astrogildo V. Oliveira Júnior}

Departamento de Educação Física e Folclore. Colégio Pedro II. Rio de Janeiro, RJ, Brasil.

\section{Brenno S. Silva}

Programa de Pós-graduação em Ciências da Atividade Física. Universidade Salgado de Oliveira. Niterói, RJ, Brasil.

\section{Daniel A. Bottino}

Laboratório de Pesquisas Clínicas e Experimentais em Biologia Vascular. Centro Biomédico.

Universidade do Estado do Rio de Janeiro. Rio de Janeiro, RJ, Brasil.

\section{Dionizio Mendes Ramos Filho}

Laboratório de Bioenergética e Fisiologia Mitocondrial. Centro de Ciências da Saúde. Universidade Federal do Rio de Janeiro. Rio de Janeiro, RJ, Brasil.

\section{Elirez B. Silva}

Laboratório de Pesquisa Clínica Escola. Departamento de Fisioterapia. Universidade Gama Filho. Rio de Janeiro, RJ, Brasil.

\section{Ercole C. Rubini}

Laboratório de Fisiologia do Exercício. Universidade Estácio de Sá. Rio de Janeiro, RJ, Brasil.

\section{Fabrício V. A. Vasconcellos}

Centro de Investigação, Formação, Inovação, Intervenção e Desporto. Faculdade de Desporto. Universidade do Porto. Porto, Portugal.

\section{Felipe A. da Cunha}

Programa de Pós-graduação em Ciências Médicas. Universidade do Estado do Rio de Janeiro. Rio de Janeiro, RJ, Brasil.

\section{Fernanda Monteiro}

Laboratório de Atividade Física e Promoção da Saúde. Instituto de Educação Física e Desportos. Universidade do Estado do Rio de Janeiro. Rio de Janeiro, RJ, Brasil.

\section{Flávia Porto}

Programa de Pós-graduação em Ciências do Exercício e do Esporte. Universidade Gama Filho. Rio de Janeiro, RJ, Brasil. 


\section{Gustavo C. Lopes}

Laboratório de Atividade Física e Promoção da Saúde. Instituto de Educação Física e Desportos. Universidade do Estado do Rio de Janeiro. Rio de Janeiro, RJ, Brasil.

\section{Jonas L. Gurgel}

Programa de Pós-graduação em Ciências

Cardiovasculares. Universidade Federal Fluminense.

Niterói, RJ, Brasil.

\section{Jonathan Myers}

VA Palo Alto Health Care System. Cardiology Division. Stanford University. Palo Alto, California, United States.

\section{Karynne Grutter}

Laboratório de Anatomia Humana. Universidade Castelo Branco. Rio de Janeiro, RJ, Brasil.

\section{Lenifran Matos-Santos}

Programa de Pós-graduação em Ciências da Atividade Física. Universidade Salgado de Oliveira. Niterói, RJ, Brasil.

\section{Lorena Paes}

Programa de Pós-graduação em Fisiopatologia Clínica e Experimental. Universidade do Estado do Rio de Janeiro. Rio de Janeiro, RJ, Brasil.

\section{Luciane P. da Costa}

Laboratório de Atividade Física e Promoção da Saúde. Instituto de Educação Física e Desportos. Universidade do Estado do Rio de Janeiro. Rio de Janeiro, RJ, Brasil.

\section{Luiz G. Kraemer-Aguiar}

Departamento de Medicina Interna. Faculdade de Ciências Médicas. Universidade do Estado do Rio de Janeiro. Rio de Janeiro, RJ, Brasil.

\section{Matheus R. Hausen}

Laboratório de Atividade Física e Promoção da Saúde. Instituto de Educação Física e Desportos. Universidade do Estado do Rio de Janeiro. Rio de Janeiro, RJ, Brasil.

\section{Paulo T. V. Farinatti}

Laboratório de Atividade Física e Promoção da Saúde. Instituto de Educação Física e Desportos. Universidade do Estado do Rio de Janeiro. Rio de Janeiro, RJ, Brasil.

\section{Rafael A. Montenegro}

Programa de Pós-graduação em Fisiopatologia Clínica e Experimental. Universidade do Estado do Rio de Janeiro. Rio de Janeiro, RJ, Brasil.

\section{Raul A. Freire}

Programa de Pós-graduação em Ciências da Atividade Física. Universidade Salgado de Oliveira. Niterói, RJ, Brasil.

\section{Renato O. Massaferri}

Programa de Pós-graduação em Ciências da Atividade Física. Universidade Salgado de Oliveira. Niterói, RJ, Brasil.

\section{Ricardo B. Oliveira}

Laboratório de Atividade Física e Promoção da Saúde. Instituto de Educação Física e Desportos. Universidade do Estado do Rio de Janeiro. Rio de Janeiro, RJ, Brasil.

\section{Ricardo G. Cordeiro}

Programa de Pós-graduação em Ciências da Atividade Física. Universidade Salgado de Oliveira. Niterói, RJ, Brasil.

\section{Sérgio Machado}

Programa de Pós-graduação em Ciências da Atividade Física. Universidade Salgado de Oliveira. Niterói, RJ, Brasil.

\section{Taciana Pinto}

Laboratório de Atividade Física e Promoção da Saúde. Instituto de Educação Física e Desportos. Universidade do Estado do Rio de Janeiro. Rio de Janeiro, RJ, Brasil.

\section{Tainah P. Lima Monteiro}

Programa de Pós-graduação em Ciências Médicas. Faculdade de Ciências Médicas. Universidade do Estado do Rio de Janeiro. Rio de Janeiro, RJ, Brasil.

\section{Tânia M. P. F. Paschoalino}

Hospital Universitário Antônio Pedro. Universidade Federal Fluminense. Niterói, RJ, Brasil.

\section{Walace D. Monteiro}

Laboratório de Atividade Física e Promoção da Saúde. Instituto de Educação Física e Desportos. Universidade do Estado do Rio de Janeiro. Rio de Janeiro, RJ, Brasil. 